\title{
Poor Prognostic Indicators of Scrub Typhus
}

\author{
Yadav SP', Yadav SK ${ }^{2}$, Kanodia P $^{3}$, Shah GS $^{4}$, Bhattarai $\mathbf{N R}^{5}$, Uranw $\mathbf{S}^{6}$
}

${ }^{1}$ Dr. Shankar Prasad Yadav, MBBS, MD, Assistant Professor, Department of Paediatrics, ${ }^{2}$ Dr. Sunil Kumar Yadav, MBBS. MD. DM, Department of Paediatrics, ${ }^{3} \mathrm{Dr}$. Piush Kanodia, MBBS, MD, DM, Department of Paediatrics, ${ }^{4} \mathrm{Dr}$. Gauri Shankar Shah, MBBS. MD. Head of Department of Paediatrics, ${ }^{5} \mathrm{Dr}$. Narayan Raj Bhattarai, Department of Clinical Microbiology, ${ }^{6} \mathrm{Dr}$. Surendra Uranw, Tropical and Infectious Disease Centre. All from the B.P.Koirala Institute of Health Sciences, Dharan, Nepal.

Address for correspondence:

Dr. Shankar Prasad Yadav

E-mail: shankardharan@gmail.com

\section{How to cite}

Yadav SP, Yadav SK, Kanodia P, Shah GS, Bhattarai NR, Uranw S. Poor Prognostic Indicators of Scrub Typhus. J Nepal Paediatr Soc 2016;36(3):314-316.

doi: http://dx.doi.org/10.3126/jnps.v36i3.15076

This work is licensed under a Creative Commons Attribution 3.0 License.

\begin{abstract}
Scrub typhus is an emerging disease in the eastern part of Nepal. Varied clinical presentation and lack of laboratory resources make diagnosis difficult. As this was the first known epidemic in this part of Nepal, unawareness resulted in late presentation with multiple complications and mortality. Hence this cases series alerts the clinician to be speculative for early diagnosis and management.
\end{abstract}

Key words: Scrub typhus, emerging, mortality

\section{Introduction}

Scrub typhus is a mite born acute, febrile, infectious illness caused by a gram negative intracellular coccobacillus Orientatsutsugamushi. The disease is distributed throughout the Asia Pacific rim and endemic in Korea, China, Taiwan, Japan, Pakistan, India, Thailand, Malaysia, and Queensland, Australia ${ }^{1}$.In Nepal, few caseshas been found in southern plain region, a study from Patan Hospital found $3.2 \%$ of 876 febrile cases serologically positive for scrub typhus ${ }^{2,3}$. While lack of easy health accessibility and diagnostic modality impose major health threat, we report five case fatalities from eastern part of Nepal. Fatality could be assigned to highly virulent infection and delayed presentation.

\section{Cases Description}

A II five cases had reported to the paediatric emergency of B.P. Koirala Institute of Health Science which is a referral institute in the eastern part of Nepal, during the monsoon season of 2015. Four were from the hilly districts and one was form the Plains. The ages were from six to fourteen years and incidentally all were female. All cases were referred from peripheral health centers after eight to ten days of illness with continuous fever of $103^{\circ} \mathrm{F}$ to $105^{\circ} \mathrm{F}$ with chills and rigors. The clinical presentationsat emergency are tabulated in Table 1.

X-ray at presentation of all the cases showed bilateral infiltrates which over 24-48 hours worsened to acute respiratory distress syndrome (Fig. 1) and the blood gas analysis showed $\mathrm{PaO}_{2} / \mathrm{FiO}_{2}<200$. Shock worsened and became catecholamine resistant, followed by pulmonary haemorrhage. The laboratory parameters at presentation of all the five cases are as depicted in Table 2. 


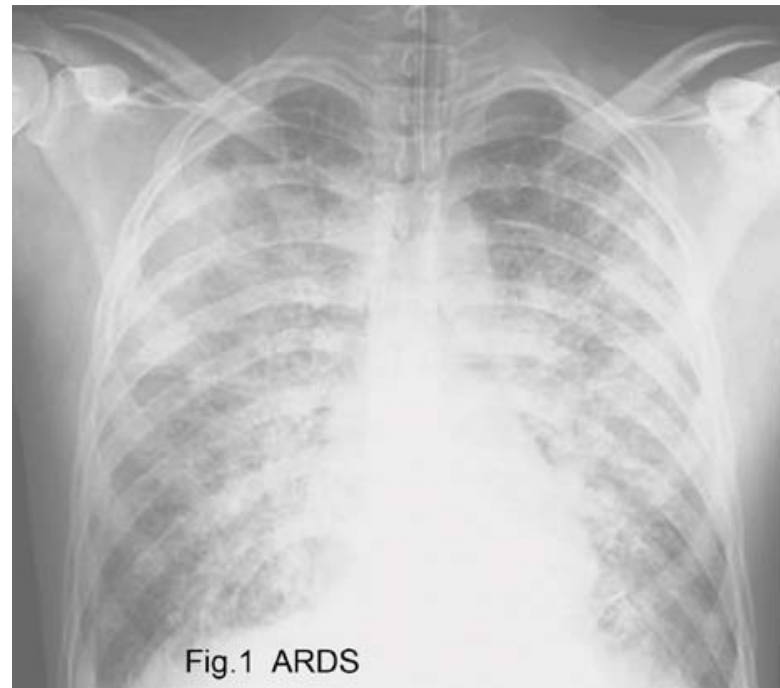

Fig 1: X-ray showed bilateral infiltrates
The majority had absence of leukocytosis with low hematocrit and severe thrombocytopenia.LDH was significantly raised along with hypoalbuminemia, raised prothrombin time (PT) and liver enzymes. Scrub typhus IgM was positive and serological tests for malaria, dengue, avian influenza, brucella and leptospira were negative. Blood culture isolated Staphylococcus aureus in one case while in other blood and urine cultures were sterile. All the cases received standard antibiotics as per hospital protocol along with doxycycline.

\section{Discussion}

Scrub typhus is one of the rare but fatal emerging diseases in Nepal. Because of lack of clinical experience many a time cases are missed at peripheral health

Table 1: Clinical features at presentation $(n=5)$

\begin{tabular}{lccc}
\hline Symptoms & No. of Patients $(\mathbf{n}=\mathbf{5})$ & Signs & No. of Patients $(\mathbf{n}=\mathbf{5})$ \\
\hline Fever (8-12 days) & 5 & Shock & 5 \\
\hline Dyspnea & 5 & Capillary leak & 5 \\
\hline Myalgia & 5 & Pedal edema & 5 \\
\hline Headache & 2 & Respiratory distress & 5 \\
\hline Pain abdomen & 2 & Hepatomegaly & 5 \\
\hline Cough & 2 & Chest crepitation & 5 \\
\hline Rashes & 1 & Maculo-papular rashes & 1 \\
\hline Drowsiness & 1 & - & 1 \\
\hline Vomiting & 1 & - & - \\
\hline
\end{tabular}

Table 2: Important laboratory parameters of all the five cases

\begin{tabular}{lccccc}
\hline Parameters & Case 1 & Case 2 & Case 3 & Case 4 & Case 5 \\
\hline TLC $/\left(\mathrm{mm}^{3}\right)$ & 4600 & 18,300 & 4700 & 5700 & 6600 \\
\hline DLC $(\%)$ & N82 L48 & N75 L20 M05 & N71 L29 & N76 L24 & N72L28 \\
\hline Hematocrit (\%) & 40 & 29.5 & 27.53 & 22.6 & 26.2 \\
\hline Platelets $/\left(\mathrm{mm}^{3}\right)$ & 10,000 & 35,000 & 30,000 & 15,000 & 27,000 \\
\hline PT(sec)/INR & $21 / 1.66$ & $25 / 2$ & $13 / 1.07$ & $20 / 1.53$ & $15 / 1.07$ \\
\hline Urea $(\mathrm{mg} / \mathrm{dl})$ & 22 & 24 & 32 & 37 & 48 \\
\hline Creatinine(mg/dl) & 0.3 & 0.4 & 0.3 & 0.7 & 0.7 \\
\hline Protein(gm/dl) & 4.9 & 4.7 & 4.8 & 4.7 & 4.4 \\
\hline Albumin(gm/dl) & 3.1 & 2.4 & 2.3 & 2.8 & 2.2 \\
\hline ALT/AST(IU/L) & $126 / 303$ & $230 / 198$ & $42 / 118$ & $126 / 303$ & $60 / 144$ \\
\hline LDH(IU/L) & 1654 & 1739 & 3384 & 2684 & 1846 \\
\hline
\end{tabular}

* TLC=Total Leucocyte Count, DLC = Differential Leucocyte Count, PT = Prothrombin Time, INR = International Normalized Ratio, ALT = Alanine Transferase, AST $=$ Aspartate Transferase, $\mathrm{LDH}=$ Lactate Dehydrogenase. 
centres. The disease presents as an acute illness with non-specific signs and symptoms ${ }^{4}$.Due to lack of characteristics eschar in $40 \%$ of the cases and unavailability of investigation modalities even at tertiary hospital, diagnosis and management is challenging ${ }^{5}$. All the cases had fever and myalgia and only once case had classical eschar. All cases had hepatomegaly but none had lymphadenopathy or spleenomegaly. Although eschar is rarely seen in cases from south east Asia $^{6}$, Iymphadenopathy is common ${ }^{7}$.Kedareshwaret al reported fever with myalgia in $80 \%$, rash in $54 \%$, with rare lymphadenopathy or spleenomegaly alike our cases $^{8}$.

The average duration of presentation at hospital of all our cases was more than 10 days, and all had developed complication in the form of plasma leakage, hepatitis, Acute Respiratory Distress Syndrome (ARDS) and refractory shock. Marked thrombocytopenia, low hematocrit, raised liver enzymes, raised LDH, and hypoalbuminemia was present in all these fatal cases. There was no case with Acute Kidney Injury.

\section{References}

1. Currie B, O'Connor L, Dwyer B. A new focus of scrub typhus in tropical Australia. Am J Trop Med Hyg 1993; 49:425.

2. Murdoch DR, Woods $\mathrm{CW}$, Zimmerman $\mathrm{MD}$ et al. The etiology of febrile illness in adults presenting to Patan Hospital in Kathmandu. NepaL. Am J Trop MedHyg2004;70(6):670-75.

3. Brown GW, Shirai A, Gan E, Bernthal P. Antibodies to typhus in Eastern Nepal. Trans Roy Soc Trop Med Hyg 1981;75:586-87.

4. Mahajan SK. Scrub typhus. J Assoc Physicians India 2005;53:954-58.

5. Silpapojakul K. Scrub typhus in the Western Pacific region. Ann Acad Med Sing1997;26:794-800.

6. Vivekanandan $M$, Mani A, Priya $Y S$, Singh AP, JayakumarS, Purty S. Outbreak of scrub typhus in Pondicherry. J Assoc Physicians India 2010;58:24-8.

7. Mahajan SK, Rolain JM, Sankhyan N, Kaushal RK, RaoultD.Pediatric scrub typhus in Indian Himalayas. Indian J Pediatr2008;75:947-49.
Multi-organ dysfunction such as AKI, ARDS, myocarditis, hepatitis, and meningo-encephalitis are common complication of scrub typhus. The occurrence of ARDS is high in scrub typhus patients who were diagnosed late and received antibiotics late ${ }^{9}$.Patients in ARDS group had more severe disease in the form of deranged liver parameters, increased serum creatinine, elevated LDH, Creatinine Phospokinase (CPK), and serum lactate ${ }^{10}$.Previous reports have shown close association of hypo albuminemia with severity of disesase $^{11}$. All the cases in our report were female. Preponderance of female getting suffered from scrub typhus is also reported byBithu $\mathrm{R}^{12}$.

\section{Conclusion}

Scrub typhus is an emerging disease with significant mortality. Non-specific symptoms and lack of diagnostic modalities heralds management challenges. Late presentation, with complications like ARDS, hepatitis, hypo-albuminemia, and shock results in mortality. Hence early clinical recognition and treatment is warranted to prevent morbidity and mortality.

8. KedareshwarPS,Narvencar, Savio R, Ramnath $P$, Nevrekar,et al. Scrub typhus in patients reporting with acute febrile illnessat a tertiary health care institution in Goa. Indian J Med Res2012;136:102024

9. Wang CC, Liu SF, Liu JW, Chung YH, Su MC, Lin MC. Acute respiratory distress syndrome in scrub typhus. Am J Trop Med Hyg 2007;76:1148-52.

10. VenkategowdaPM,RaoSM,Mutkule DP, RaoMV,Taggu AN. Scrub typhus: Clinical spectrum and outcome. Indian J Crit Care Med 2015;19(4):208-13.

11. Sridevi S, Mythuli SV, ManjulaDevi AJ, Shanthi B, KalaiSelvi VS. Hpoalbuminemia and multi organ dysfunction in a case of scrub typhus. JCase Rep 2013;3(1):180-182

12. Bithu R, Kanodia V, Maheshwari RK. Possibility of scrub typhus in fever of unknown origin (FUO) cases: An experience from Rajasthan. Indian J Med Microbio/2014;32:387-90. 